\title{
OPTIMAL LOCATION FOR ELICITING THE TIBIAL H- REFLEX AND MOTOR RESPONSE
}

\author{
Mustafa Görkem ÖZYURT PhD, Mohammed SHABSOG MD, Merve DURSUN MD and Kemal S. TÜRKER \\ BDS, $\mathrm{PhD}$ \\ School of Medicine, Koç University, Istanbul, Turkey \\ Corresponding author: Kemal S. Türker (kturker@ku.edu.tr) \\ Tel: +902123381174 \\ Fax: +902123381165
}

Running Title: Targeted Nerve Stimulation

\section{Ethical Publication Statement}

We confirm that we have read the Journal's position on issues involved in ethical publication and affirm that this report is consistent with those guidelines.

\section{Acknowledgments}

We are grateful to our subjects who volunteered for this project. We also acknowledge Koç University School of Medicine for generous funding this lab. Additionally, we thank Ata Berk Demir, Damla Erbil and Süleyman Savran who helped us during data collection and Beatrice Selen Şenocak who improved the English of the manuscript. None of the authors has any conflict of interest to disclose.

This article has been accepted for publication and undergone full peer review but has not been through the copyediting, typesetting, pagination and proofreading process, which may lead to differences between this version and the Version of Record. Please cite this article as doi: 10.1002/mus.26308 


\title{
OPTIMAL CATHODE LOCATION TO ELICIT H- REFLEX AND MOTOR RESPONSE IN SOLEUS MUSCLE
}

\begin{abstract}
Introduction: Although there are numerous protocols to adjust the amplitude of the H-reflex relative to the size of the direct motor response (M-response), the optimal stimulating location has not been described. We aimed to determine the optimal positioning of the stimulating cathode when evoking the tibial nerve H-reflex and Mresponse.

Methods: A small cathode was placed on defined points above the popliteal fossa while an anode was fixed on the patella. The tibial nerve was stimulated electrically and the response of the soleus muscle was recorded using intramuscular and surface electromyography.

Results: We found that more lateral points along a line drawn across the popliteal fossa were the best locations to obtain the M-only response while stimulating the midpoint was optimal for obtaining only the H-reflex.

Discussion: Using specified locations for electrical stimulation to evoke H-reflex and M-response, the functionality of the tibial nerve can be assessed.
\end{abstract}


Keywords: H-reflex, direct motor response, soleus muscle, electrical stimulation, cathode localization

\section{Introduction}

Studying spinal cord circuits allows us to understand the wiring of neurons that control our motor behavior. Sensory neuron-motor neuron interactions and the related interneuron circuits can be studied by various experimental tools. The electrical equivalent of the monosynaptic spindle reflex, the Hoffmann reflex (H-reflex), is one of the most convenient tools to extract information about these circuits. More specifically, the H-reflex can be used to study synaptic modulation ${ }^{1}$, short-term learning ${ }^{2}$, common drive to motor neurons ${ }^{3}$, and many other neuronal and neuromuscular phenomena ${ }^{4,5}$ In addition, the H-reflex can be used to assess several neurological disorders clinically, including spasticity and denervation ${ }^{6-8}$. Operant conditioning with the tibial H-reflex recently becomes an attractive therapeutic tool in chronic spinal cord injury to improve recovery of locomotion ${ }^{9}$.

When a motor fiber is stimulated, its orthodromic activity evokes a direct motor response (M-response) from the muscle. The orthodromic activation of the spindle afferents, on the other hand, elicits the H-reflex ${ }^{10,11}$. Obtaining an isolated H-reflex (i.e. without the M-response) is necessary for the study of spindle input to motor neurons ${ }^{12-14}$. Eliciting the opposite - an M-response without the H-reflex -is required to study Renshaw circuitry ${ }^{15}$.

Eiciting the H-reflex and the M-response from the soleus muscle usually is achieved by stimulating the tibial nerve in the popliteal fossa. Since both the common peroneal and he tibial nerves can be stimulated in the popliteal fossa ${ }^{16}$, ${ }^{17}$, positioning of the stimulating electrodes needs to be done carefully. Furthermore, the surface electromyography (SEMG) record of the muscle of interest can be contaminated by cross-talk from other muscles that become active upon stimulation ${ }^{17,}{ }^{18}$. This degree of cross-talk can be assessed by using intramuscular electrodes in addition to SEMG.

Two methods have been developed to standardize the study of the H-reflex: amplitude normalization and stimulus normalization. The amplitude normalization method requires that the size of the H-reflex be quantified as a percentage of the maximal M-response ${ }^{19,20}$ while the stimulus normalization method is performed so that the stimulus intensity used to evoke the H-reflex is adjusted to generate the maximal M-response ${ }^{4,21}$. 
In this study, we introduce a third methodof standardization when studying the H-reflex and M-response. We refer to our method as the "cathode positioning method". I

\section{Methods}

The Committee on Human Research at Koç University approved the procedure in accordance with the Declaration of Helsinki. All the experiments were performed in the neurophysiology laboratory of Koç University on subjects who signed informed consent. Healthy subjects, between 20-30 years, were recruited from Koç University School of Medicine. Exclusion criteria were neurological disorders, the use of any medication, or back pain at the time of the study. Ten subjects ( 8 male and 2 female) participated in this study and all experiments were performed on the right leg.

Setup: A CED 1902 Quad System MKIII amplifier and CED Power 1401 MKII DAC were used for recordings. Spike2 7.03 software was used to perform data analysis (Cambridge Electronic Design, England).

Electromyography: To record the electrical activity of the soleus muscle, both surface and intramuscular EMG were used. SEMG was recorded with a sampling rate of 2,000 Hz and filtered with a 20-500 $\mathrm{Hz}$ band pass filter. Intramuscular EMG was recorded with a 20,000 Hz sampling rate and filtered with a 100-5,000 Hz band pass filter. Two of the standard SEMG electrodes $(\mathrm{Ag} / \mathrm{AgCl})$ were placed on the soleus muscle after rubbing the skin with sandpaper, cleaning with alcohol and applying electrode gel to decrease the impedance of the skin. The soleus muscle was identified by palpation while the subject performed plantar flexion. SEMG electrodes were placed over the lower one-third of the muscle on the posterolateral leg. The electrodes were placed $4 \mathrm{~cm}$ apart ${ }^{22}$ and attached to the skin with surgical tape. For intramuscular EMG, silver fine-wire electrodes coated with Teflon (core diameter of $75 \mu \mathrm{m}$; Medwire, USA) were used. The wires were stripped of their Teflon coating for $3 \mathrm{~mm}$ at their tips so they could function as multi motor unit (MMU) recording wires, allowing them to record from a larger area of the muscle. The sterile bipolar intramuscular electrodes were inserted into the muscles via a 25 -gauge surgical needle. The needle was inserted into the muscle belly at a point in between the two SEMG electrodes and removed immediately, leaving a pair of fish-hooked fine wires inside the muscle. For grounding, a sterile hook-shaped silver lip clip was attached to the lip in contact with mouth mucosa ${ }^{23}$. 
Experimental procedure: The subject rested prone on a bed-like platform and was asked to relax his/her leg muscles throughout the experiment, which lasted about two hours. Just after placement of the intramuscular and SEMG electrodes, the stimulation points on the popliteal fossa were drawn using marker. First, the skin crease at the point of knee flexion was identified and the midpoint was determined and a "crease line" was drawn. In addition, we wished to investigate the responses of the muscle when the nerve is stimulated close to its origin where the sciatic nerve branches into the tibial nerve. After examining human cadavers, we defined the upper line ("proximal line") as $3 \mathrm{~cm}$ above the crease line. This line corresponds to a position of the tibial nerve just distal to the bifurcation point. Although the distance between the bifurcation point and the crease would vary according to the height of the subjects, this variation would be small as the subjects' leg lengths were between 60 and $64 \mathrm{~cm}$ (from the crease to lumbar spine). The midpoint (i.e. point 0 ) of both lines intersected with an imaginary vertical line originating from the Achilles' tendon (Figure 1). The side-to-side distance between the most lateral and medial part of the leg was approximately $16 \mathrm{~cm}$ for the crease and $18 \mathrm{~cm}$ for the proximal line.

After determination of the side-to-side distance between the most lateral and medial part of the leg, we marked four equally-spaced points along each side of the midpoint of each line. The points that were lateral to the midpoint were labeled with positive numbers $(+1,+2,+3,+4)$ and the points that were medial to the midpoint were labeled with negative numbers $(-1,-2,-3,-4)$. In total, 18 points were marked ( 9 along the proximal line and 9 along the crease line). The minimum current at the midpoint of the crease line that elicited an unambiguous H-reflex on the intramuscular EMG record was defined as the threshold stimulus intensity. We then used a stimulus intensity of $20 \%$ above the threshold intensity at each point of stimulations. The order in which the points were stimulated was determined randomly in order to avoid anticipatory effects, which are believed to influence the H-reflex ${ }^{24}$.

A constant current stimulator (Digitimer DS7A) was used to stimulate the tibial nerve. The anode $(10 \times 12 \mathrm{~cm})$ was placed immediately proximal to the patella and the cathode $(3 \times 3 \mathrm{~mm})$ was placed on one of the eighteen points along the two lines that were drawn across the popliteal fossa. After placement of both stimulating electrodes, an elastic band, which covered the circumference of the knee, was used to fix these electrodes in position. Since this bandage was elastic, it applied similar pressure to all the electrodes. We used monopolar stimulation of the tibial nerve, ${ }^{25,26}$ fixing the position of the anode over the patella and moving the position of the cathode in the popliteal 
fossa to elicit a response. A total of 25 stimuli ( $1 \mathrm{~ms}$ pulse width and interstimulus interval 0.8 to 1.2 seconds) were applied to each of the marked points, with each point being stimulated separately.

Evaluation: The size of the H-reflex and M-response was defined as the peak-to-peak amplitude of the unrectified EMG record. Since the stimulation at each stimulus intensity activates a fixed number of motor axons, the resulting M-response can be used for normalizing these responses, thus making it possible to make an inter-subject comparison. Hence, each recording was normalized according to the maximal M-response of that trial.

We averaged responses of 25 stimuli for each point for both M-response and H-reflex separately. After that, all 18 points were normalized using the maximum M-response. This normalization was done separately for MMU and SEMG records, for which distinct maximum M-responses were determined. Lastly, each normalized coordinate was also averaged among all 10 subjects and determined as a percentage. All the amplitudes in the results are indicated as the mean amplitude of the 10 subjects. Among those averaged values, two-way ANOVA was used to determine the difference of the responses of all 18 individual points in both lines. Multiple comparisons were corrected using Tukey's method. The level of significance was selected as $p<0.05$. All statistical analysis was performed using GraphPad 6.

\section{Results}

Height and weight were $175-190 \mathrm{~cm}$ and $75-90 \mathrm{~kg}$ for men, and $160-170 \mathrm{~cm}$ and $50-60 \mathrm{~kg}$ for women (BMI19 to 23). The M-response activity evoked upon stimulation of the lateral points $(+2,+3)$ was strong, but was barely 
detectable upon stimulation of the medial points along both lines. The amplitude of the M-responses was similar in both intramuscular and SEMG recordings. While the highest M-response amplitude was consistently recorded upon stimulation of the +2 and +3 points along the crease line (Figure 2), the maximum H-reflex amplitude was recorded upon stimulation of the midpoint of the crease line. Furthermore, the mean amplitude of the H-reflex elicited from stimulating points along the crease line was significantly larger than that obtained from stimulation of points along the proximal line.

\subsection{H-reflex}

Intramuscular EMG: The greatest mean amplitude was $73 \%$ of the maximum M-response. This response was obtained from stimulating at the midpoint of the crease line and was significantly greater in amplitude than that obtained from stimulating at the midpoint of the proximal line $(\mathrm{p}<0.0001)$. The amplitude of the reflex obtained from stimulation at the midpoint of the crease line was twice that of the response resulting from stimulation at point $+1(\mathrm{p}=0.0365)$. Nonetheless, stimulation of the midpoint and of point +1 along the crease line, resulted in responses that were larger than those elicited by stimulation at the other points.

Stimulation at points $0,+1$, and +2 along the proximal line also reliably evoked the H-reflex. Stimulation at the midpoint of the crease line resulted in a significantly greater H-reflex amplitude than did stimulation at the midpoint of the proximal line $(\mathrm{p}<0.0001)$.

$\underline{\text { SEMG: }}$ The greatest mean H-reflex amplitude that was recorded by SEMG was elicited by stimulating the midpoint of the crease line and was $40 \%$ of the maximum M-response. Stimulation at the midpoint and at point +1 along the crease line induced a greater H-reflex response than did stimulation at the other points along this line. This finding is in agreement with the results from the intramuscular EMG record. Stimulation at points $0,+1,+2$ and +3 along the proximal line resulted in similar H-reflex amplitudes that were larger than the responses elicited from stimulation at other points along the proximal line.

This article is protected by copyright. All rights reserved 


\subsection{M-response}

Intramuscularly and superficially recorded M-response sizes were well correlated with each other. Stimulation at points +2 and +3 along both the crease and proximal lines resulted in an M-response with a greater amplitude, compared to that of responses obtained from stimulating the other points along both lines. In addition, the Mresponses with the greatest amplitudes were seen upon stimulation of point +2 along the crease line. Furthermore, slight M-response amplitudes were detected by stimulating at points -1 and +4 along at the crease line. With regards to the proximal line, the largest average M-response was also recorded upon stimulation at the +2 point. However, due to the high variability in the mean amplitudes of M-responses recorded when stimulating along the proximal line, no significant stimulus location-dependent difference was observed (for MMU; +2 vs +3 : $\mathrm{p}=0.3783,+2$ vs +4 : $\mathrm{p}=0.2517$; for SEMG; $-1 \mathrm{vs}+2: \mathrm{p}=0.2071,+2$ vs $+4: \mathrm{p}=0.4763$ ).

\subsection{Ideal M-response and H-reflex positions}

When stimulating along the crease line, intramuscular recordings revealed that the H-reflex was significantly greater compared to the M-response when the cathode was placed at the midpoint $(\mathrm{p}=0.001)$. In contrast, there was no such exact position for eliciting the H-reflex by stimulating along the proximal line. The best locations to stimulate in order to elicit an M-response with minimum H-reflex contamination were point +2 (For MMU; $\mathrm{p}=0.0028$, SEMG; $\mathrm{p}<0.0001$ ) and +3 along the crease line (For MMU; $\mathrm{p}=0.0017$, SEMG; $\mathrm{p}<0.0001$ ). Also, stimulation at points +2 and

+3 gave almost identical results in terms of the amplitude of the M-response. However, a similar conclusion about the optimal cathode position could not be made for the proximal line, although point +2 appears to be the optimal position to stimulate in order to elicit a pure M-response.

This article is protected by copyright. All rights reserved 


\section{Discussion}

To improve the accuracy and reliability of the assessment of the sensory and motor nerve conditions in clinics, we suggest that optimal locations should be used for electrical stimulation of the tibial nerve through popliteal fossa to obtain pure H-reflex and M-response.

The majority of protocols for studying the H-reflex and M-response in the literature are based on using specific techniques to determine the optimal location for electrical nerve stimulation using physiological tools. The most widely used methods involve stimulus-evoked EMG responses ${ }^{27-30}$ or twitch recording ${ }^{31,32}$. These protocols involve placing the EMG electrodes on the surface of a muscle and/or linking a force transducer to the innervated limb for twitch recording. The optimal cathode position is defined as the point where the minimal stimulus intensity evokes an observable response in the SEMG record or the force record ${ }^{33}$.

The biggest problem in the above-mentioned protocols is the issue of cross-talk. When a region is electrically stimulated with the purpose of activating a muscle of interest, nerves that activate other muscles and/or the antagonist muscle can also be stimulated and result in non-specific twitch responses, H-reflexes or M-responses, especially at higher stimulus intensities ${ }^{18}$. The use of intramuscular EMG, multi-differential SEMG electrodes ${ }^{34}$ and specific location of the stimulating cathode as tested in this study might minimize cross-talk and help the experimenter determine the optimal point for stimulation. Hence, in this study, we used surface and intramuscular EMG to determine the optimal location of the cathode for goal-dependent electrical nerve stimulation.

Optimal cathode position for eliciting an isolated H-reflex: In the literature, to induce the H-reflex in the soleus muscle either bipolar stimulation, where both anode and cathode are placed on the popliteal fossa ${ }^{35}$ or monopolar stimulation, where the anode is fixed just above the patella and the cathode is placed on the popliteal fossa ${ }^{36}$ were used. However, the exact position of the cathode has not been described in either case. In the current study, the optimal point of stimulation for eliciting the isolated H-reflex was found to be the midpoint of the crease line.

Optimal cathode position for eliciting an isolated M-response: the optimal location of stimulus delivery for selective stimulation of the motor fibers was the second major finding in this study. Stimulation of the more lateral points along the crease line, especially of points +2 and +3 resulted in the largest M-responses with minimal H-reflex contamination. The results of both surface and intramuscular EMG support this conclusion. However, since the 
lateral points along the crease line are close to the common peroneal nerve, stimulating at the more medial point (i.e. point +2 ) may be desirable to limit the possibility of activating the tibialis anterior muscle. As stimulation at both points +2 and +3 evoked similar responses, point +2 along the crease line can be defined as the best location to stimulate in order to obtain an M-response from the soleus with minimum H-reflex and tibialis anterior activity.

The proposed localization technique can be used in a variety of clinical and basic research settings. To study circuitries such as recurrent inhibition, stimulations that generate pure M-response are preferred ${ }^{15}$ and these can be achieved using the methodology described in this article. In a clinical setting, assessment of the integrity of specific neuronal pathways ${ }^{37,38}$, for example, the neuronal network of the spindle primary afferents ${ }^{9}$, can be achieved using electrical stimulation intensities that generate only the H-reflex response using the protocol described in this paper.

\section{Abbreviations}

- EMG: Electromyography

- H-reflex: Hoffmann reflex

- $\mathrm{M}_{\max }$ : Maximum direct motor response

- MMU: Multi motor unit

- M-response: Direct motor response 
- SEMG: Surface electromyography

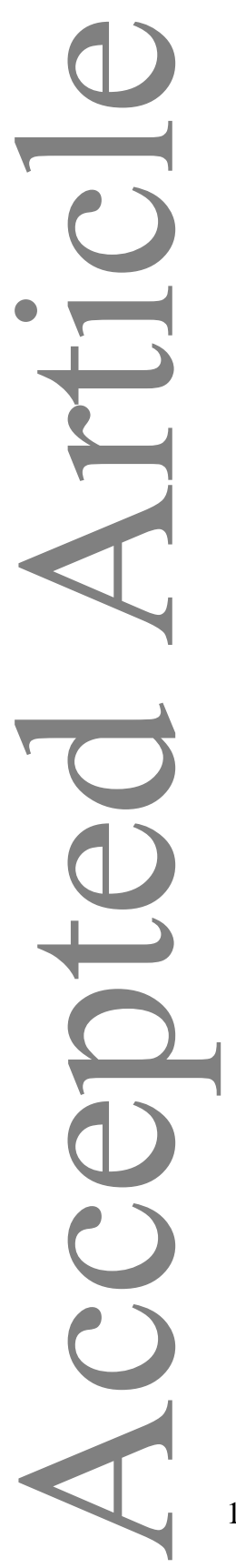

\section{References}

1. Stein RB, Capaday C. The modulation of human reflexes during functional motor tasks. Trends Neurosci 1988;11(7):328-32.

2. Wolpaw JR. The complex structure of a simple memory. Trends Neurosci 1997;20(12):588-94. 
3. Laine CM, Yavuz SU, Farina D. Task-related changes in sensorimotor integration influence the common synaptic input to motor neurones. Acta Physiol (Oxf) 2014;211(1):229-39.

4. Niazi IK, Turker KS, Flavel S, Kinget M, Duehr J, Haavik H. Changes in H-reflex and V-waves following spinal manipulation. Exp Brain Res 2015;233(4):1165-73.

5. Palmieri RM, Ingersoll CD, Hoffman MA. The Hoffmann Reflex: Methodologic Considerations and Applications for Use in Sports Medicine and Athletic Training Research. Journal of Athletic Training 2004;39(3):268-277.

6. Burke D. Clinical uses of H reflexes of upper and lower limb muscles. Clinical Neurophysiology Practice 2016;1:9-17.

7. Yang JF, Fung J, Edamura M, Blunt R, Stein RB, Barbeau H. H-Reflex Modulation During Walking in Spastic Paretic Subjects. Canadian Journal of Neurological Sciences / Journal Canadien des Sciences Neurologiques 2015;18(4):443-452.

8. Mazzini L, Balzarini C, Gareri F, Brigatti M. H-reflex changes in the course of amyotrophic lateral sclerosis. Electroencephalogr Clin Neurophysiol 1997;104(5):411-7.

9. Thompson AK, Pomerantz F, Wolpaw JR. Operant conditioning of a spinal reflex can improve locomotion after spinal cord injury in humans. J Neurosci 2013;33(6):2365-2375.

10. Katz R, Pierrot-Deseilligny E. Recurrent inhibition in humans. Prog Neurobiol 1999;57(3):325-355.

11. Piotrkiewicz M, Kudina L, Mierzejewska J. Recurrent inhibition of human firing motoneurons (experimental and modeling study). Biol Cybern 2004;91(4):243-57.

12. Baldissera F, Bellani G, Cavallari P, Lalli S. Changes in the excitability of the H-reflex in wrist flexors related to the prone or supine position of the forearm in man. Neurosci Lett 2000;295(3):105-8.

13. Hoffmann P. Beitrag zur Kenntnis der menschlichen Reflexe mit besonderer Berucksichtigung der elektrischen Erscheinungen. Arch Anat Physiol 1910;1:223-246.

14. Matthews WB. Ratio of maximum $\mathrm{H}$ reflex to maximum M response as a measure of spasticity. $J$ Neurol Neurosurg Psychiatry 1966;29(3):201-204.

15. Kudina LP, Pantseva RE. Recurrent inhibition of firing motoneurones in man. Electroencephalogr Clin Neurophysiol 1988;69(2):179-85. 
16. Shefner JM, Logigian EL. Conduction velocity in motor, cutaneous afferent, and muscle afferent fibers within the same mixed nerve. Muscle Nerve 1994;17(7):773-8.

17. Netter FH. 1987. The CIBA collection of medical illustrations: Musculoskeletal system. Anatomy, physiology and metabolic disorders. CIBA-GEIGY Corporation.

18. Turker KS, Miles TS. Cross-talk from other muscles can contaminate EMG signals in reflex studies of the human leg. Neurosci Lett 1990;111(1-2):164-9.

19. Hultborn H, Illert M, Nielsen J, Paul A, Ballegaard M, Wiese H. On the mechanism of the post-activation depression of the H-reflex in human subjects. Exp Brain Res 1996;108(3):450-62.

20. Pierrot-Deseilligny E, Mazevet D. The monosynaptic reflex: a tool to investigate motor control in humans. Interest and limits. Neurophysiol Clin 2000;30(2):67-80.

21. Brinkworth RS, Tuncer M, Tucker KJ, Jaberzadeh S, Turker KS. Standardization of H-reflex analyses. $J$ Neurosci Methods 2007;162(1-2):1-7.

22. Tucker KJ, Turker KS. A new method to estimate signal cancellation in the human maximal M-wave. $J$ Neurosci Methods 2005;149(1):31-41.

23. Turker KS, Miles TS, Le HT. The lip-clip: a simple, low-impedance ground electrode for use in human electrophysiology. Brain Res Bull 1988;21(1):139-41.

24. Brunia CHM. Neural aspects of anticipatory behavior. Acta Psychologica 1999;101(2-3):213-242.

25. Wee AS, Leis AA, Kuhn AR, Gilbert RW. Anodal block: can this occur during routine nerve conduction studies? Electromyogr Clin Neurophysiol 2000;40(7):387-91.

26. Vuckovic A, Tosato M, Struijk JJ. A comparative study of three techniques for diameter selective fiber activation in the vagal nerve: anodal block, depolarizing prepulses and slowly rising pulses. J Neural Eng 2008;5(3):275-86.

27. Botter A, Vieira TM. Optimization of surface electrodes location for H-reflex recordings in soleus muscle. J Electromyogr Kinesiol 2017;34:14-23.

28. Dishman JD, Cunningham BM, Burke J. Comparison of tibial nerve H-reflex excitability after cervical and lumbar spine manipulation. Journal of Manipulative and Physiological Therapeutics 2002;25(5):318-325.

29. Funase K, Miles TS. Observations on the variability of the $\mathrm{H}$ reflex in human soleus. Muscle Nerve 1999;22(3):341-6. 
30. Hugon M. 1973. Methodology of the Hoffmann Reflex in Man. Vol. 3. 277-293.

31. Dean JC, Collins DF. Nonlinear twitch torque summation by motor units activated at M-wave and H-reflex latencies. Muscle Nerve 2009;40(2):221-30.

32. Folland JP, Wakamatsu T, Fimland MS. The influence of maximal isometric activity on twitch and Hreflex potentiation, and quadriceps femoris performance. Eur J Appl Physiol 2008;104(4):739.

33. Crenna P, Frigo C. Excitability of the soleus H-reflex arc during walking and stepping in man. Exp Brain Res 1987;66(1):49-60.

34. Koh TJ, Grabiner MD. Evaluation of methods to minimize cross talk in surface electromyography. $J$ Biomech 1993;26 Suppl 1:151-7.

35. Cheng J, Brooke JD, Misiaszek JE, Staines WR. The relationship between the kinematics of passive movement, the stretch of extensor muscles of the leg and the change induced in the gain of the soleus $\mathrm{H}$ reflex in humans. Brain Res 1995;672(1-2):89-96.

36. Hultborn H, Pierrot-Deseilligny E. Changes in recurrent inhibition during voluntary soleus contractions in man studied by an H-reflex technique. J Physiol 1979;297(0):229-51.

37. Lowrey CR, Bent LR. Modulation of the soleus H-reflex following galvanic vestibular stimulation and cutaneous stimulation in prone human subjects. Muscle Nerve 2009;40(2):213-20.

38. Raynor EM, Shefner JM. Recurrent inhibition is decreased in patients with amyotrophic lateral sclerosis. Neurology 1994;44(11):2148-53.

\section{Figure Legends}

Figure 1. The location of the defined points drawn on the popliteal fossa and placement of recording electrodes on soleus. Figure on the left indicates the crease line and the proximal line that were separated by $3 \mathrm{~cm}$. 
Along each line, there are 9 points. The midpoint of each line (point 0 ) was determined with reference to the Achilles' tendon. Points that are lateral with respect to the midpoint are specified with a "+" sign, whereas points that are medial with respect to the midpoint are specified with a "-" sign. Figure on the right shows the position of the surface and intramuscular electromyography recording electrodes which detect the responses simultaneously during stimulation at the defined points in the popliteal fossa.

Figure 2. The recorded results by surface and intramuscular electromyography (EMG) upon electrical stimulation of the defined points. Amplitudes obtained from the identified locations (from -4 to +4 ) are shown as waveforms (figures on top) and as columns with error bars (figures on the bottom). The figures on the top show the obtained responses as waveforms depending on the point of stimulation, whereas the column graphs on the bottom present the results of the statistical analyses conducted on these responses. The amplitudes of the responses were normalized to the maximum direct motor response $\left(\mathrm{M}_{\max }\right)$ and averaged. Both the surface electromyography (SEMG) and the multi motor unit (MMU) records indicated that the midpoint and point +1 of both lines were the optimal points for obtaining the Hoffmann reflex (H-reflex). Also, stimulation at lateral $(+)$ points along the crease line evoked significantly larger direct motor responses (M-responses), except at point +4 . Stimulation of points along the proximal line did not seem to be advantageous for eliciting the M-response or H-reflex. Neither the Mresponse nor the H-reflex was significantly greater in amplitude when evoked by stimulation at points along the proximal line (Error bars show standard error, ${ }^{*} \mathrm{p}<0.05$, $* * \mathrm{p}<0.01, * * * \mathrm{p}<0.001, * * * * \mathrm{p}<0.0001$ ). 

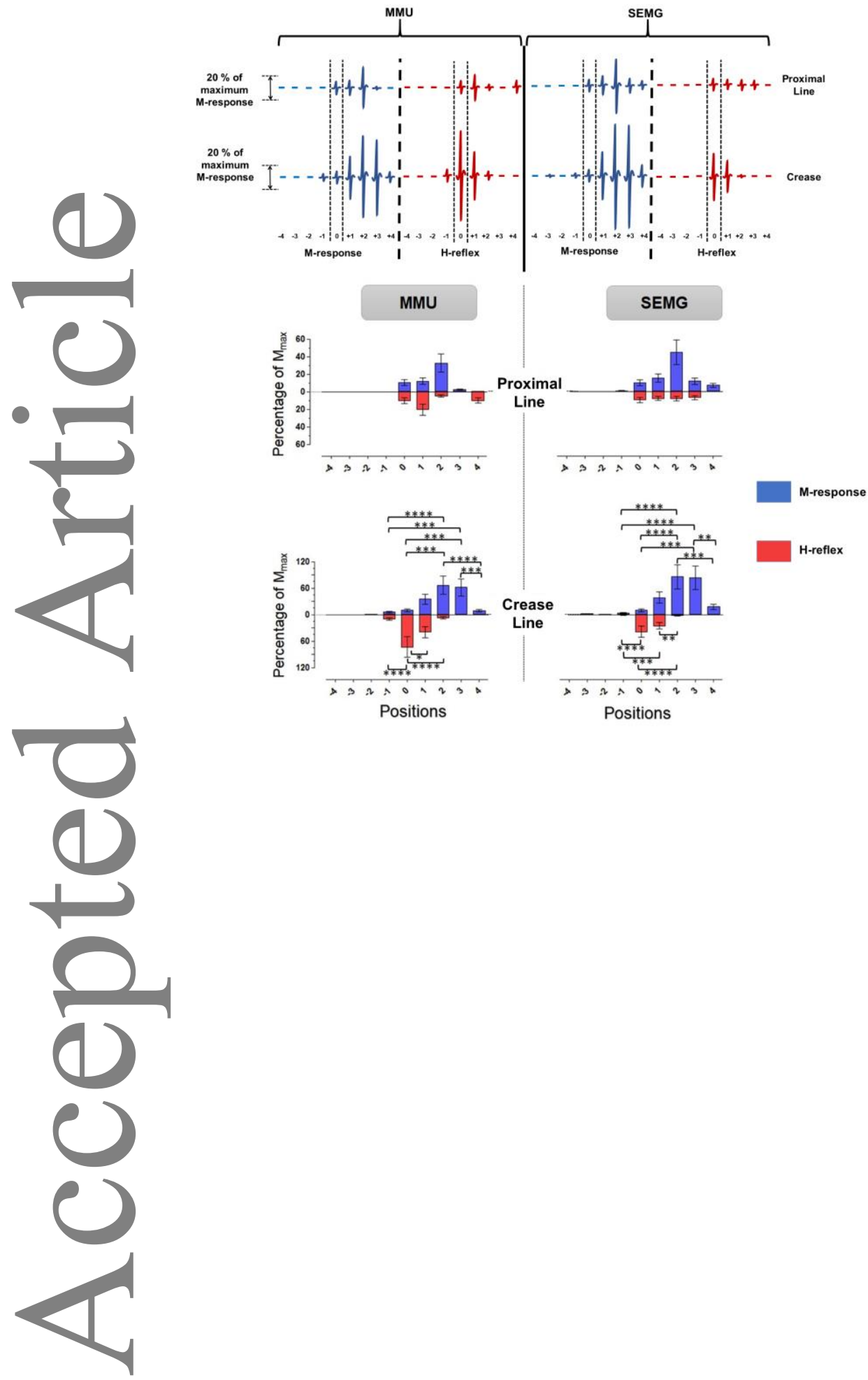

This article is protected by copyright. All rights reserved 